\title{
The Status of the Distance Scale ${ }^{1}$
}

\author{
G. A. Tammann, B. R. Parodi, B. Reindl \\ Astronomisches Institut der Universität Basel, Venusstr. 7, CH-4102 \\ Binningen, Switzerland
}

\begin{abstract}
Cepheids are the pillar of the extragalactic distance scale, but their reach in distance is not sufficient to calibrate $H_{0}$. Yet HST has provided Cepheid distances to eight galaxies which have produced SNe Ia. The latter are used as nearly perfect standard candles to carry the distance scale to $30000 \mathrm{~km} \mathrm{~s}^{-1}$, giving $H_{0}=58 \pm 5$. This determination finds support in other methods, but its range in distance and its weight is unparalleled.
\end{abstract}

\section{Introduction}

The determination of large scale distances and hence the calibration of the Hubble constant $H_{0}$ has been fundamentally influenced by the Hubble Space Telescope (HST). For the first time this extremely powerful instrument has made accessible a simple, reliable, and clear-cut method to reach into the cosmic expansion field by calibrating the luminosity of type Ia supernovae ( $\mathrm{SNe}$ Ia) - the best standard candles known - through Cepheid distances of their nearest representatives. The dominant rôle of Cepheids on this route to $H_{0}$ has presumably prompted the organizers of this Colloquium to ask for a status report on the (distant) distance scale. The Cepheid surveys to be reported here subtend only tiny angles in the sky, but they comprise large parts of, or even entire galaxies, and in this sense may also justify the Colloquium's emphasis on "Large-Scale Surveys".

In Section 2 we discuss Cepheids as distance indicators and quote the Cepheid distances of eight galaxies which have produced nine SNe Ia. The Cepheid-calibrated SNe Ia luminosities are given in Section 3. In Section 4 we discuss the Hubble diagram of an objective sample of blue SNe Ia and homogenize the SNe Ia relative to decline rate $\Delta m_{15}$ and color $B-V$. The data in Sections 3 and 4 are combined in Section 5 to derive the large-scale value of $H_{0}$. The result is compared with external evidence in Section 6 . Some conclusions follow in Section 7.

${ }^{1}$ A report on the "HST SNe Ia Calibration Program for the Hubble Constant" by A. Sandage, A. Saha, L. Labhardt, N. Panagia, F. D Macchetto, \& G. A. Tammann) 


\section{Cepheids as Distance Indicators}

Cepheids are presently, through their period-luminosity $(P-L)$ relation, the most reliable and least controversial distance indicators. The slope and the zero-point of the $P-L$ relation is taken from the very well observed Cepheids in the Large Magellanic Cloud (LMC), whose distance modulus is adopted to be $(m-M)=18.50$ (Madore \& Freedman 1991).

An old $P-L$ relation calibrated by Galactic Cepheids in open clusters, and now vindicated by Hipparcos data (Sandage \& Tammann 1998), gave $(m-M)_{\text {LMC }}=18.59$ (Sandage \& Tammann 1968, 1971). Hipparcos data combined with more modern Cepheid data give an even somewhat higher modulus (Feast \& Catch pole 1997). Reviews of Cepheid distances (Federspiel, Tammann, \& Sandage 1998; Gratton 1998; Feast 1999; Walker 1999a) cluster around $18.56 \pm 0.05,-a$ value in perfect agreement with the purely geometrical distance determination of SN 1987A (18.58 \pm 0.05; Gilmozzi \& Panagia 1999; Sonneborn et al. 1997; Walker 1999a). From RR Lyr stars Gratton (1998) concludes that $(m-M)_{\mathrm{LMC}}=18.54 \pm 0.12$.

A general problem of distance determinations is that distance indicators are occasionally used which have never been demonstrated to be useful and reliable. Their application relies on the assumption of uniformity. Examples are statistical parallaxes of Galactic RR Lyrae stars, which are sensitive to the sample selection (Walker 1999b), and red-giant clump stars, whose luminosity is expected to be metal- and age-dependent (Chaboyer 1999). These untested methods yield typically small LMC distances and have led to what has recently been termed the "short distance scale". The corresponding LMC distances carry low weight.

There has been much debate about the possibility that the $P-L$ relation of Cepheids depends on metallicity. The question here is more of principal than of practical importance because the mean metallicity of the seven spiral galaxies and one Am galaxy considered below hardly differs by much from the Galactic metallicity. Direct observational evidence for a (very) weak metallicity dependence comes from the fact that the metal-rich Galactic Cepheids give perfectly reasonable distances for both the moderately metal-poor LMC Cepheids and the really metal-poor SMC Cepheids and, still more importantly, that their relative distances are wavelength-independent (Di Benedetto 1997; cf. Tammann 1997). Much progress has been made on the theoretical front. Saio \& Gautschy (1998) and Baraffe et al. (1998) have evolved Cepheids through the different crossings of the instability strip and have investigated the pulsational behavior at any point. The resulting (highly metal-insensitive) $P-L$ relations in bolometric light have been transformed into $P-L$ relations at different wavelengths by means of detailed atmospheric models; the conclusion is that any metallicity dependence of the $P-L$ relations is negligible (Sandage, Bell, \& Tripicco 1998; Alibert et al. 1999; cf. however Bono, Marconi, \& Stellingwerf 1998, who strongly depend on the treatment of stellar convection).

While remaining uncertainties of the $P-L$ relation and the zero-point seem to have only minor practical consequences, the application to HST observations is by no means simple. The photometric zero-point, the linearity over the field, crowding, and cosmic rays raise technical problems. The quality of the derived distances depends further on (variable) internal absorption and the number of 
Table 1. Absolute $B, V$, and $I$ magnitudes of blue SNe Ia calibrated through Cepheid distances of their parent galaxies. (Errors in $0{ }^{\mathrm{m}} 01 \mathrm{in}$ parentheses)

\begin{tabular}{lcccccccc}
\hline SN & Rem. & $\log v$ & $(m-M)_{B}$ & $(m-M)_{V}$ & $M_{B}^{0}$ & $M_{V}^{0}$ & $M_{I}^{0}$ & $\Delta m_{15}$ \\
\hline $1895 \mathrm{~B}$ & 1 & 2.464 & $28.13(08)$ & $28.10(07)-19.87(22)$ & $\ldots$ & $\ldots$ & $\ldots$ \\
$1937 \mathrm{C}$ & 2 & 2.519 & $28.36(09)$ & $28.36(12)$ & $-19.56(15)-19.54(17)$ & $\ldots$ & $0.87(10)$ \\
$1960 \mathrm{~F}$ & 3 & 3.072 & $31.16(10)$ & $31.13(10)$ & $-19.56(18)-19.62(22)$ & $\ldots$ & $1.06(12)$ \\
$1972 \mathrm{E}$ & 4 & 2.464 & $28.13(08)$ & $28.10(07)$ & $-19.64(16)-19.61(17)$ & $-19.27(20)$ & $0.87(10)$ \\
$1974 \mathrm{G}$ & 5 & 2.820 & $31.46(17)^{a}$ & $\ldots$ & $-19.67(34)-19.69(27)$ & $\ldots$ & $1.11(06)$ \\
$1981 \mathrm{~B}$ & 6 & 3.072 & $31.10(05)^{a}$ & $\ldots$ & $-19.50(14)-19.50(10)$ & $\ldots$ & $1.10(07)$ \\
$1989 \mathrm{~B}$ & 7 & 2.734 & $30.22(12)^{a}$ & $\ldots$ & $-19.47(18)-19.42(16)$ & $-19.21(14)$ & $1.31(07)$ \\
$1990 \mathrm{~N}$ & 8 & 3.072 & $32.03(22)^{a}$ & $\ldots$ & $-19.39(26)-19.41(24)$ & $-19.14(23)$ & $1.03(05)$ \\
$1998 \mathrm{bu}$ & 9 & 2.814 & $30.37(16)^{a}$ & $\ldots$ & $-19.76(31)-19.69(26)-19.43(21)$ & $1.08(05)$ \\
\hline \multicolumn{7}{c}{ mean (straight, excl. SN 1895B) } & $-19.57(04)-19.56(04)-19.26(06)$ \\
\hline \multicolumn{7}{c}{ mean (weighted, excl. SN 1895B) } & $-19.55(07)-19.53(06)$ & $-19.25(09)$ \\
\hline
\end{tabular}

a The true distance modulus is listed.

Remarks AND REFERENCES: (1) in NGC 5253 - Saha et al. 1995; (2) in IC 4182 Saha et al. 1994; (3) in NGC 4496A - Saha et al. 1996b; (4) in NGC 5253 - Saha et al. 1995 (5) in NGC 4414 - Turner et al. 1998; (6) in NGC 4536 - Saha et al. 1996a; (7) in NGC 3627 - Saha et al. 1999; (8) in NGC 4639 - Saha et al. 1997; (9) in NGC 3368 - Tanvir et al. 1995.

available Cepheids in view of the finite width of the instability strip. (An attempt to beat the latter problem by using a $P-L$-color relation is invalid because the underlying assumption of constant slope of the constant-period lines is unjustified; cf. Saio \& Gautschy 1998). Typical errors of individual Cepheid distances from HST are therefore $\pm 0.2 \mathrm{mag}$ ( $10 \%$ in distance). For four of the nine SNe la in Table 1 the resulting errors in luminosity are smaller, because they suffer (small) absorption similar to "their" Cepheids, such that only apparent distance moduli are needed.

The nine SNe la in the eight galaxies with known Cepheid distances from HST are listed in Table 1. Six galaxies are from the "HST SNe Ia Calibration Program for the Hubble Constant"; two additional ones are taken from the literature. The distance moduli are based on an adopted, conservative value of $(m-M)_{\text {LMC }}=18.50$.

The Cepheids observed with the wide-field camera of HST (WFP2) are corrected by $+00^{\mathrm{m}} 05$ for the photometric short exposure/long exposure zero-point effect of that camera (Stetson et al. 1998; Saha et al. 1995).

The Cepheid distances in Table 1 have been re-analyzed by Gibson et al. (1999). While their object-to-object photometry corroborates the original sources to within a few percent, their treatment of Cepheid reddening and sample selection is unacceptable, leading to distance moduli $0^{\mathrm{m}} 13$ smaller on average. The prize is that their resulting SNe Ia luminosities have a noticeably larger scatter $\left(\sigma_{B}=0^{\mathrm{m}} 16\right.$ instead of $\left.\sigma_{B}=0^{\mathrm{m}} 12\right)$. Yet the most important point is 
that their adopted mean SNe Ia luminosities are fainter than those in Table 1 by only $0.05,0.07$, and 0.15 (the latter value from only four SNe Ia) in $B, V$, and $I$, respectively. These minute differences are even below the statistical errors.

On the other hand a re-analysis of NGC 4414 (Turner et al. 1998) with an independent photometry package (ROMAPHOT) suggests that the $B$ magnitudes were measured to be $\sim 0$. 10 too bright (Thim 2000).

The most severe problem of the Cepheid distances is selection bias. The intrinsic width of the instability strip poses the problem that at any given period a fair number of bright and faint Cepheids must be sampled. But at shorter periods the faint counterparts are progressively discriminated against. This leads to a systematic underestimate of the distance (Sandage 1988). The effect becomes apparent if sufficient numbers of Cepheids are available because the derived distance increases as one goes to longer periods. Narasimha \& Mazumdar (1998) and Mazumdar \& Narasimha (2000) suggest that in the case of NGC 4321 (M 100) (Ferrarese et al. 1996) the distance was underestimated by $\sim 15 \%$ due to bias. The distance moduli in Table 1 were derived with the Sandage bias in mind, but too few Cepheids are available for a rigid treatment. In principle the bias problem remains that Cepheid distances tend to be underestimates if one works near the detection limit. The severity of the bias decreases as the available period interval increases.

\section{The Calibration of SNe Ia Luminosities from Cepheid Distances}

SNe Ia in galaxies with Cepheid distances are shown in Table 1. The adopted apparent maximum magnitudes of the SNe la as well as the internal absorption of the last five entries in Table 1 are detailed elsewhere (Parodi et al. 1999). The straight and weighted means of the eight adopted SNe Ia (SN 1895B is excluded because it has no $M_{V}(\max )$ ) are also shown in Table 1.

The r.m.s. deviation of a single $M$-value in Table 1 amounts to only $\sigma_{B}=\sigma_{V}=0^{\mathrm{m}} 12$ confirming the notion of blue SNe Ia being exceptionally useful standard candles. In fact, the deviations are smaller than the estimated errors of the Cepheid distances. This means that the Cepheid distances are better than expected, and there is little room for distance errors due to metallicity differences.

The empirical luminosity calibration is in perfect agreement with presently available theoretical models. Höflich \& Khokhlov (1996) have fitted sufficiently blue models, i.e. $(B-V)<0.2$ at maximum, to the light curves and spectra of $16 \mathrm{SNe}$ Ia. Their mean luminosity in $B$ and $V$ is only 0.05 fainter than in Table 1. In a recent review Branch (1998) has concluded that present theory is best satisfied by $M_{\mathrm{B}} \approx M_{\mathrm{V}}=-19.4$ to -19.5 for blue SNe Ia.

Della Valle et al. (1998) have attempted to derive an independent distance of NGC 1380, host of SN $1992 \mathrm{~A}$, by means of the peak of the luminosity function of globular clusters (GCLF) and advocated a low luminosity of the SN. However, the GCLF method is known to give sometimes erratic results (Tammann \& Sandage 1998).

Lennicutt, Mould, \& Freedman (1998), and Freedman (1999) have discarded several of the calibrators in Table 1 and added two that are not based on direct Cepheid distances to the host galaxy. They have consequently derived 
a fainter mean absolute $B$ magnitude than in Table 1 . Specifically, they assume that the distance of the early-type galaxies NGC 1316 and NGC 1380 in the Fornax cluster, parent galaxies of SN $1980 \mathrm{~N}$ and SN $1992 \mathrm{~A}$, are identical with that of the spiral NGC 1365 for which a Cepheid distance is available. Suntzeff et al. (1999) have also considered the questionable SN 1980N and SN 1992A as possible calibrators. However, with the significantly larger Cepheid distance of another Fornax spiral (NGC1425; Mould et al. 1999) this has become untenable.

The HST project for the luminosity calibration of SNe Ia will be continued. Cepheid observations are presently granted for NGC 4527 (with the peculiarspectrum and possibly overluminous SN 1991T) and NGC 3982 (with SN 1998aq).

\section{The Hubble Diagram of Distant SNeIa}

A complete sample of $45 \mathrm{SNe}$ Ia has been collected from the literature, the majority from the Calán/Tololo survey (Hamuy et al. 1996). They fulfil the condition that their values $m_{B}(\max )$ and $m_{V}(\max )$ are available; wherever $m_{I}(\max )$ is known it is retained. They must have occurred after 1985 to ensure the quality of their photometry, and they must have recession velocities of $v_{220}>1000 \mathrm{~km} \mathrm{~s}^{-1}$ (after correction for Virgocentric streaming velocities) to guard against excessive effects of peculiar velocities. Finally they must be blue, i.e. $\left(B_{\max }-V_{\max }\right) \leq 0.20$; this is to exclude peculiar SNe Ia, which are known to be red and underluminous, as well as SNe Ia which suffer much absorption in their parent galaxies. All SNe Ia are corrected for Galactic absorption (Schlegel, Finkbeiner, \& Davis 1998).

The SNe Ia of the sample, for which spectra are known, are all "Branch normal" (Branch, Fisher, \& Nugent 1993). The only exceptions are SN 1991T and 1995ac; they are probably overluminous and excluded here. Only seven SNe Ia of the sample have $(B-V)>0.06$. They are underluminous judging from their velocity distance, and five of them lie close to the center of their parent spiral galaxies. Their redness is probably due to internal absorption. They are left out in the following. Had they been retained on the assumption that their color is intrinsic, the derived value of $H_{0}$ would become somewhat lower. If they were retained and corrected for standard absorption they would have an unnoticeable effect on $H_{0}$ as derived below.

The remaining sample of $36 \mathrm{SNe}$ Ia has a small range in color of $-0.10 \leq(B-V) \leq 0.06$ and a mean color of $\langle B-V\rangle=-0.010 \pm 0.09$. The mean color of the SNe Ia in E/S0 galaxies, of the outlying SNe Ia in spirals, and of the calibrators in Table 1 - which are expected to suffer minimum internal absorption or are corrected for absorption - is $\langle B-V\rangle=-0.014 \pm 0.011$. The close agreement of these two mean colors is a convincing demonstration that the sample of $36 \mathrm{SNe}$ Ia is essentially free of internal absorption. This is not surprising because unreddened SNe Ia have at large distances a considerably higher discovery chance.

The $36 \mathrm{SNe}$ Ia define tight Hubble diagrams in $B, V$, and $I$. The scatter is only $\sigma=0.23,0.22$, and 0.18 , respectively.

Blue SNe Ia at maximum phase still show some variation in temperature, spectrum, and presumably $\mathrm{Ni}$ mass. It is hence indicated to ask whether these parameters correlate with luminosity. However, data on the relevant parameters 
are too sparse to establish a firm relation. Instead it is reasonable to seek for empirical correlations between luminosity and directly observable "second parameters" like decline rate $\Delta m_{15}$ (i.e. the luminosity decrease in $B$ magnitudes within 15 days after $B$ maxim um), SN color, Hubble type of the parent galaxy, and radial distance from the center of the parent galaxy.

In fact a luminosity dependence on $\Delta m_{15}$, SN color $B-V$, and Hubble type $T$ does exist. However, an increase of the luminosity scatter with decreasing galactocentric distance (Wang, Höflich, \& Wheeler 1997) is not confirmed by blue SNe Ia.

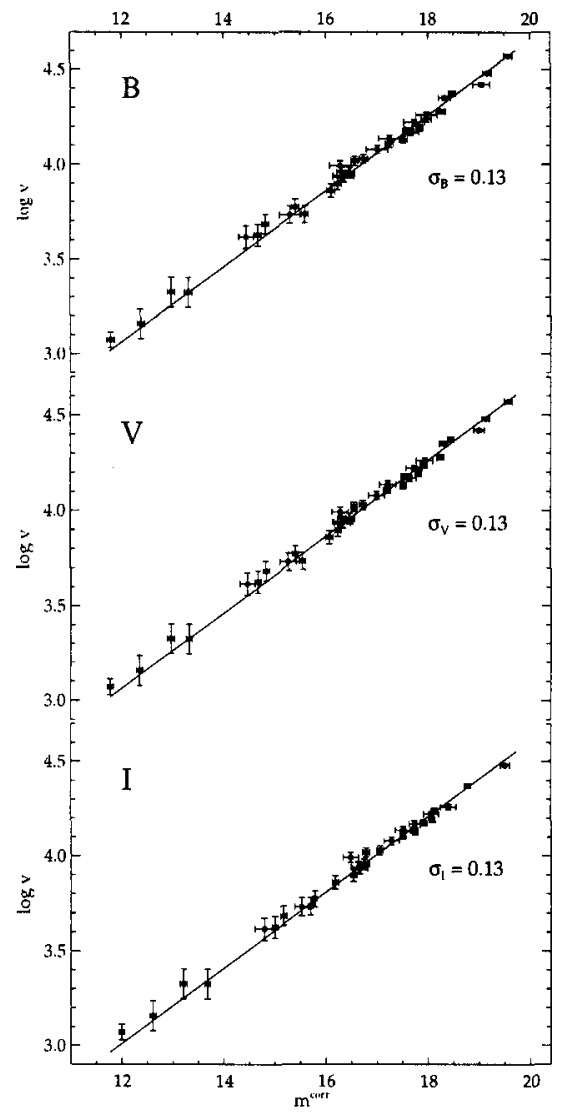

Figure 1. The Hubble diagram in $B, V$, and $I$ for 36 blue SNe Ia. The fitted lines have slope 0.2 ; they only consider SNe Ia with $v>10000 \mathrm{~km} \mathrm{~s}^{-1}$. The velocities are corrected for a self-consistent Virgocentric infall model (Kraan-Korteweg 1986) if $v<3000 \mathrm{~km} \mathrm{~s}^{-1}$; for $v>3000 \mathrm{~km} \mathrm{~s}^{-1}$ an additional correction was applied for the motion of $630 \mathrm{~km} \mathrm{~s}^{-1}$ relative to the CMB dipole anisotropy (Smoot et al. 1992). - (From Parodi et al. 1999).

To obtain quantitative relations between the maximum magnitudes and any of the second parameters, one must correlate the residuals (read in mag- 
nitude) from the mean Hubble line with slope 0.2 with $\Delta m_{15}, B-V$, and $T$. However, there are strong reasons to believe that the residuals of SNe Ia inside $10000 \mathrm{~km} \mathrm{~s}^{-1}$ are not only caused by luminosity variations, but also by deviation from a pure Hubble flow. If only the SNe Ia with $v>10000 \mathrm{~km} \mathrm{~s}^{-1}$ are considered, one finds

$$
\begin{aligned}
B^{\text {corr }} & =B-0.34\left(\Delta m_{15}-1.1\right)-2.45(B-V), \\
V^{\text {corr }} & =V-0.41\left(\Delta m_{15}-1.1\right)-1.41(B-V), \\
I^{\text {corr }} & =I-0.43\left(\Delta m_{15}-1.1\right)-1.37(B-V) .
\end{aligned}
$$

When these equations are applied to the $36 \mathrm{SNeIa}$ of the sample one obtains homogenized magnitudes $m^{\text {corr }}$ as if all SNe Ia had $\Delta m_{15}=1.10$ and $B-V=0.00$. The second parameters $\Delta m_{15}$ and $(B-V)$ are nearly orthogonal to each other, but the corrected magnitudes show no remaining dependence on Hubble type $T$. (SNe Ia in $\mathrm{E} / \mathrm{S} 0$ galaxies are on average fainter than those in spirals, but they are also faster decliners).

The 36 corrected SNe Ia define Hubble diagrams in $B, V$, and $I$ as shown in Fig. 1. A fit to the SNela with $v>10000 \mathrm{~km} \mathrm{~s}^{-1}$ gives:

$$
\begin{array}{lll}
\log v=0.2 m_{B}^{\text {corr }}+(0.660 \pm 0.006) ; & \sigma_{\mathrm{B}}=0.13, & N=19 \\
\log v=0.2 m_{V}^{\text {corr }}+(0.663 \pm 0.006) ; & \sigma_{\mathrm{V}}=0.13, & N=19 \\
\log v=0.2 m_{I}^{\text {corr }}+(0.610 \pm 0.007) ; & \sigma_{\mathrm{I}}=0.13, & N=14 .
\end{array}
$$

The scatter in magnitude of $\sigma=0.13$ is now impressively small, in fact it could be caused entirely by observational error. The intrinsic luminosity scatter of homogenized SNe Ia is therefore below the present detection limit.

The scatter of the nearer SNe Ia with $v<10000 \mathrm{~km} \mathrm{~s}^{-1}$ is somewhat larger, i.e. $\sigma=0.16$. This must be due to peculiar motions in the order of $\Delta v / v \approx 0.05$, which is most reasonable considering our local motion of $630 \mathrm{~km} \mathrm{~s}^{-1}$ with respect to the CMB. There is also a suggestion of the nearer SNe Ia to lie somewhat above the mean Hubble line, which seems to suggest a slightly higher local value of $H_{0}$ (see below).

\section{5. $H_{0}$ from $\mathrm{SNe} I a$}

Simple transformation of equations (4-6) leads to

$$
\begin{aligned}
& \log H_{0}=0.2 M_{B}^{\text {corr }}+(5.660 \pm 0.006), \\
& \log H_{0}=0.2 M_{V}^{\text {corr }}+(5.663 \pm 0.006), \quad \text { and } \\
& \log H_{0}=0.2 M_{I}^{\text {corr }}+(5.610 \pm 0.007) .
\end{aligned}
$$

Here only the absolute magnitudes $M_{i}$ of the calibrators in Table 1 must be inserted to obtain $H_{0}$. But the calibrators must also be corrected by equations $(1-3)$, giving $M_{B}^{\text {corr }}=-19.50 \pm 0.08, M_{V}^{\text {corr }}=-19.50 \pm 0.07$, and $M_{I}^{\text {corr }}=-19.21 \pm 0.10$. Inserting these values into equations $(7-9)$ yields $H_{0}(B)=57.5 \pm 2.9, H_{0}(V)=57.9 \pm 2.7$, and $H_{0}(I)=58.6 \pm 3.6$. The weighted mean of these three values is

$$
H_{0}=57.9 \pm 1.7 \quad \text { (statistical error). }
$$




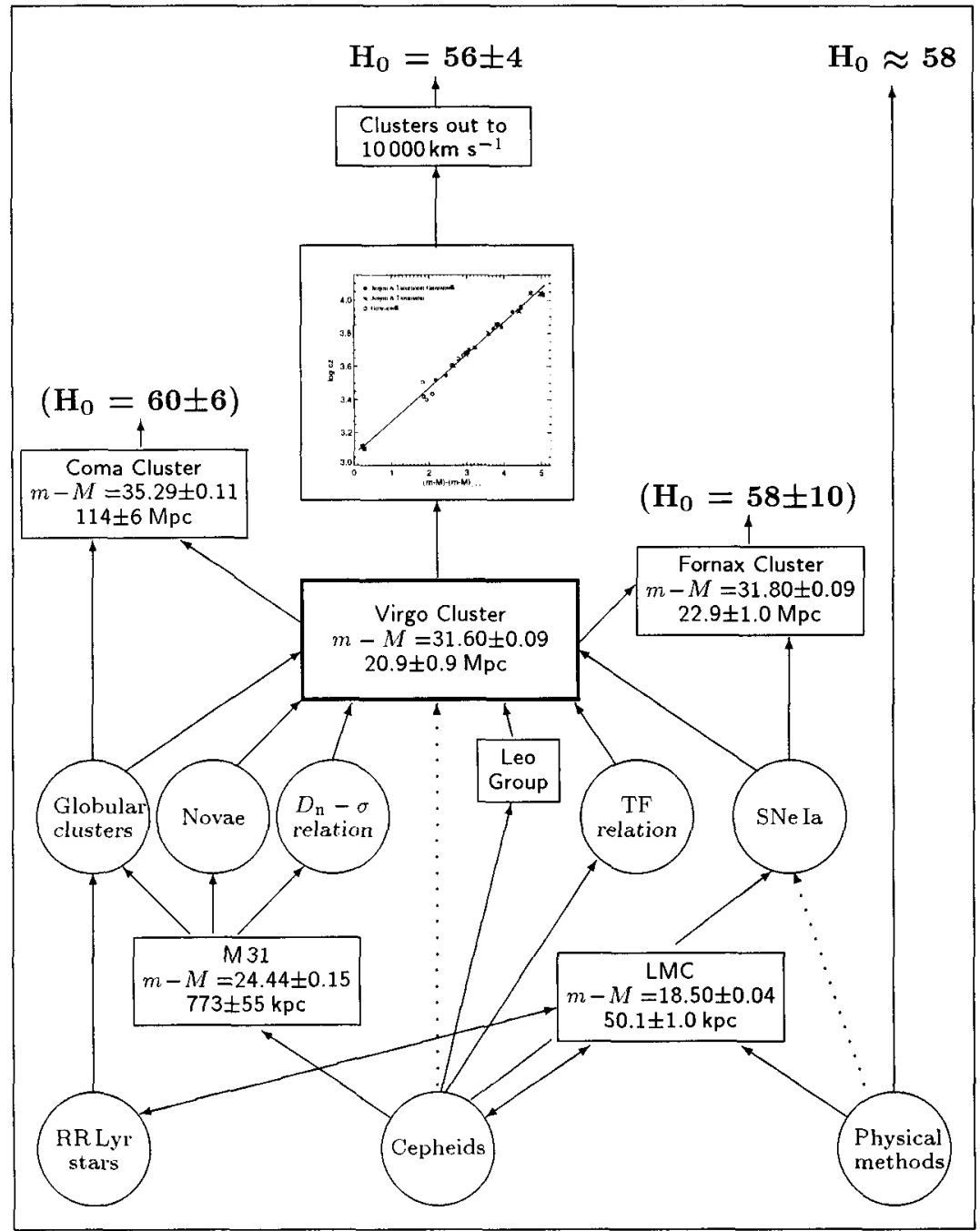

Figure 2. Schematic presentation of the distance scale based on various determinations of the Virgo cluster distance. 
The external errors will be discussed in Section 6 .

Had we not corrected the sample of distant $\mathrm{SNe}$ Ia and the calibrators by equations $(1-3)$, the result would have been $H_{0}=55.0 \pm 1.6$. The reason for the lower value is that the calibrators must have Cepheids in their parent galaxies, which hence are of late type, and SNe Ia in late-type galaxies are brighter (and have lower $\Delta m_{15}$ ) than their counterparts in E/S0 galaxies. The corrections for the decline rate have sometimes been exaggerated in the past (Phillips 1993; Hamuy et al. 1996; Suntzeff et al. 1999; Gibson et al. 1999) leading to larger values of $H_{0}$. However, these larger decline rate corrections rest on absolute magnitudes from unreliable distance indicators or on residuals from the Hubble line where peculiar motions are still not negligible. The corrections in equations (1-3), based only on distant SNe Ia with $v>10000 \mathrm{~km} \mathrm{~s}^{-1}$ remove all luminosity dependence of the SNe Ia on $\Delta m_{15}$ and $B-V$. Any larger $\Delta m_{15}$ corrections would introduce a luminosity dependence of opposite sign.

It may be noted that the coefficients of the color term in equations $(1-3)$ are significantly smaller than the ones expected from a standard absorption/reddening ratio. It is therefore likely that the dependence of luminosity on color is due to an intrinsic effect. The color corrections significantly decrease the scatter about the Hubble line. They do not influence, however, the value of $H_{0}$ because the calibrators and the 36 sample SNe Ia have closely the same color.

The value of $H_{0}$ in equation (10) holds only for SNe Ia with $v>10000 \mathrm{~km} \mathrm{~s}^{-1}$, i.e. over very large scales. The 17 SNe Ia with $1000 \mathrm{~km} \mathrm{~s}^{-1}<v<10000 \mathrm{~km} \mathrm{~s}^{-1}$ give instead a mean value of $H_{0}=61.1 \pm 2.1$, suggesting a local, yet rather large volume of lower mean density and with a slight overexpansion rate of $\sim 5 \%$ (Tammann 1998; Zehavi et al. 1998). Combining the evidence from $B, V$, and $I$ it is at present a $2-3 \sigma$ effect. Newly discovered SNe Ia will improve on the variance of the expansion field.

\section{6. $H_{0}$ from External Evidence}

The available space does not allow a thorough comparison of $H_{0}$ in equation (10) with independent evidence. Therefore only an overview of other distance scales, leading mainly through the Virgo cluster, is given in Fig. 2. The reader finds a more detailed discussion in Tammann, Sandage, \& Reindl (1999). A compilation of present results of physical methods, i.e. the Sunyaev-Zeldovich effect, gravitational lenses, and CMB fluctuations, can be found in, e.g., Tammann (1999; see also Lasenby et al. 1999). About $75 \%$ of the distance scale in Fig. 2 rests on the local calibration through Cepheids. It is therefore not truly independent of the SNe Ia.

The route to $H_{0}$ through field galaxies is not satisfactory, because it will never reach out to $10000 \mathrm{~km} \mathrm{~s}^{-1}$ and beyond. In addition, the route is technically difficult because of selection effects, causing the galaxies which are missed in a sample to be almost as important as those which constitute the sample. In spite of this, careful treatment of the Malmquist bias leads to values of $H_{0}=55-60$ at distances of $1000-5000 \mathrm{~km} \mathrm{~s}^{-1}$ (Sandage 1996a,b, 1999; Theureau et al. 1997; Goodwin, Gribbin, \& Hendry 1997; Federspiel 1999). Values as high as $H_{0}=70$ are an unfailing indication that the bias problem has been mishandled. 


\section{Conclusions}

Group and outside the Virgo cluster yield $H_{0}=65 \pm 4$. But reaching out to barely $1100 \mathrm{~km} \mathrm{~s}^{-1}$ this value has no cosmological significance. It is therefore mandatory to extend the distance scale.

By far the most reliable way to extend the distance scale is by SNe Ia which are, after standardization as to decline rate and color, unparalleled standard candles. The number of Cepheid-calibrated and distant SNe Ia out to $30000 \mathrm{~km} \mathrm{~s}^{-1}$ is now sufficiently large to make the statistical error of $H_{0}$ negligible (cf. equation 7$)$.

Sources of systematic errors are:

(1) the adopted zero-point of $(m-M)_{\mathrm{LMC}}=18.50$, a value which is likely to be too small by $0{ }^{\mathrm{m}} 06 \pm 0 .{ }^{\mathrm{m}} 10$. This will reduce $H_{0}$ by $3 \pm 5 \%$.

(2) The selection effect against faint Cepheids at the detection limit (Sandage 1988) may systematically underestimate the distances in Table 1 by $5 \pm 5 \%$ with a. corresponding reduction of $H_{0}$.

(3) Remaining small metallicity effects of the $P-L$ relation will probably not change the calibration by more than $0.06(3 \%)$.

(4) The coefficients of the $\Delta m_{15}$-term in equations 1-3 carry a statistical error of 0.2 ; increasing (decreasing) the coefficients by so much increases (decreases) $H_{0}$ by 2.7 units $(5 \%)$.

(5) The nearly perfect agreement of the me an color of the calibrators and the obviously-unreddened $\mathrm{SNe} \mathrm{Ia}$ on the one hand, and the remaining distant SNe Ia on the other hand (cf. Section 4), make it improbable that the latter still carry an unaccounted mean reddening of more than 0.02 ; about half of the corresponding absorption is automatically corrected for by the color term in equations (1-3); the other half can increase $H_{0}$ by $2 \%$ at most.

Compounding the systematic errors and adding them to equation (7) leads to a most probable value of

$$
H_{0}=57.9_{-8.3}^{+4.2}
$$

Acknowledgments. The authors thank the Swiss National Science Foundation for financial support.

\section{References}

Alibert, Y., Baraffe, I., Hauschildt, P., \& Allard, F. 1999, A\&A, 344, 551

Baraffe, I., Alibert, Y., Méra, D., Chabrier, G., \& Beaulieu, J.-P. 1998, ApJ, 499, L205

Bono, G., Marconi, M., \& Stellingwerf, R. F. 1998, ApJS, 122, 167

Branch, D. 1998, ARA\&A, 36, 17

Branch, D., Fisher, A., \& Nugent, P. 1993, AJ, 106, 2383

Chaboyer, B. 1999, private communication

Della Valle, M., Kissler-Patig, M., Danziger, J., \& Storm, J. 1998, MNRAS, 299, 267 
Di Benedetto, G. P. 1997, ApJ, 486, 60

Feast, M. 1999, PASP, 111, 775

Feast, M. \& Catchpole, R. M. 1997, MNRAS, 286, L1

Federspiel, M. 1999, Ph.D. Thesis, Univ. of Basel

Federspiel, M., Tammann, G. A., \& Sandage, A. 1998, ApJ, 495, 115

Ferrarese, L., Freedman, W. L., Hill, R. J., et al. 1996, ApJ, 464, 568

Freedman, W. L. 1999, BAAS, 194, 3909

Gibson, B. K., Stetson, P. B., Freedman, W. L., et al. 1999, ApJ, in press [astro-ph/9908149]

Gilmozzi, R. \& Panagia, N. 1999, Space Telescope Science Inst. Preprint Series No. 1319

Goodwin, S. P., Gribbin, J., \& Hendry, M. A. 1997, AJ, 114, 2212

Gratton, R. 1998, in 19th Texas Symposium on Relativistic Astrophysics and Cosmology, in press

Hamuy, M., Phillips, M. M., Maza, J., Suntzeff, N. B., Schommer, R. A., \& Aviles, R. 1996, AJ, 112, 2408

Höflich, P. \& Khokhlov, A. 1996, ApJ, 457, 500

Kennicutt, R. C., Mould, R. J., \& Freedman, W. L. 1998, preprint

Kraan-Korteweg, R. 1986, A\&AS, 66, 255

Lasenby, A., Bridle, S.. Hobson, M., \& Efstathiou, G. 1999, in 19th Texas Symposium on Relativistic Astrophysics and Cosmology, preprint

Madore, B. F. \& Freedman, W. L. 1991, PASP, 103, 933

Mazumdar, A. \& Narasimha, D. 2000, in these proceedings, p. 229

Mould, J. R., Huchra, J. P., Freedman, W. L., et al. 1999, ApJ, in press [astro$\mathrm{ph} / 9909260]$

Narasimha, D. \& Mazumdar, A. 1998, astro-ph/9803195

Parodi, B. R., Saha, A., Sandage, A., \& Tammann, G. A. 1999, ApJ, submitted Phillips, M. M. 1993, ApJ, 413, L105

Saha, A., Labhardt, L., Schwengeler, H., Macchetto, F. D., Panagia, N., Sandage, A., \& Tammann, G. A. 1994, Ap.J, 425, 14

Saha, A., Sandage, A., Labhardt, L., Schwengeler, H., Tammann, G. A., Panagia, N., \& Macchetto, F. D. 1995, ApJ, 438, 8

Saha, A., Sandage, A., Labhardt, L., Tammann, G. A., Macchetto, F. D., \& Panagia, N. 1996a, ApJ, 466, 55

Saha, A., Sandage, A., Labhardt, L., Tammann, G. A., Macchetto, F. D., \& Panagia, N. 1996b, ApJS107, 693

Saha, A., Sandage, A., Labhardt, L., Tammann, G. A., Macchetto, F. D., \& Panagia, N. 1997, ApJ, 486, 1

Saha, A., Sandage, A., Labhardt, L., Tammann, G. A., Macchetto, F. D., \& Panagia, N. 1999, ApJ, 522, 802

Saio, H. \& Gautschy, A. 1998, ApJ, 498, 360

Sandage, A. 1988, PASP, 100, 935

Sandage, A. 1996a, AJ, 111, 1 
Sandage, A. 1996b, AJ, 111, 18

Sandage, A. 1999, preprint

Sandage, A., Bell, R. A., \& Tripicco, M. J. 1998, ApJ, 522, 250

Sandage, A. \& Tammann, G. A. 1968, ApJ, 151, 531

Sandage, A. \& Tammann, G. A. 1971, ApJ, 167, 293

Sandage, A. \& Tammann, G. A. 1998, MNRAS, 293, L23

Schlegel, D., Finkbeiner, D., \& Davis, M. 1998, ApJ, 500, 525.

Smoot, G., Bennett, C. L., Kogut, A., et al. 1992, ApJ, 396, L1

Sonneborn, G., Fransson, C., Lundquist, P., et al. 1997, ApJ, 477, 848

Stetson, P. B., Saha, A., Ferrarese, L., et al. 1998, ApJ, 508, 491

Suntzeff, N. B., Phillips, M. M., Covarrubias, R., et al. 1999, AJ, 117, 1175

Tammann, G. A. 1997, J. Astrophys. Astro., 18, 271

Tammann, G. A. 1998, in General Relativity, 8th Marcel Grossmann Symp., ed. T. Piran (Singapore: World Scientific), 243

Tammann, G. A. 1999, in Dark Matter in Astrophysics and Particle Physics, ed. H. V. Klapdor-Kleingrothaus \& L. Baudis (Bristol: Inst. Physics Publ.), 153 , preprint

Tammann, G. A. \& Sandage, A. 1999, in ASP Conf. Ser. Vol. 167, Harmonizing the Cosmic Distance Scale in a Post-Hipparcos Era, ed. D. Egret \& A. Heck (San Francisco: ASP), 204

Tammann, G. A., Sandage, A., \& Reindl, B. 1999, in 19th Texas Symposium on Relativistic Astrophysics and Cosmology, preprint [astro-ph/9904360]

Tanvir, N. R., Shanks, T., Ferguson, H. C., \& Robinson, D. R. T. 1995, Nature, 377,27

Theureau, G., Hanski, M., Ekholm, T., et al. 1997, A\&A, 322, 730

Thim, F. 1999, in these proceedings, p. 231

Turner, A., Ferrarese, L., Saha, A., et al. 1998, ApJ, 505, 207

Walker, A. R. 1999a, in Post-Hipparcos Cosmic Candles, ed. A. Heck \& F. Caputo (Dordrecht: Kluwer), 125

Walker, A. R. 1999b, private communication

Wang, L., Höflich, P., \& Wheeler, J. C. 1997, ApJ, 483, L29

Zehavi, I., Riess, A. G., Kirshner, R. P., \& Dekel, A. 1998, ApJ, 503, 483 